\title{
Cloud Computing: Concepts, Architecture and Applied Research
}

\author{
Yingjie Wang ${ }^{1-2, a}$ \\ ${ }^{1}$ College of Information Engineering, Dalian University, Dalian 116622, P . R. China \\ ${ }^{2}$ Software School, Dalian University of Technology, Dalian 116621, P . R. China \\ ayingjiewang2008@163.com
}

Keywords: Cloud Computing, Service Models, Deployment Models

Abstract. Since cloud computing could be understood only by specialists, it is necessary to write a clear but not complex description regarding knowledge to promote common people understanding this technology. This paper refined three essential characteristics of cloud computing, analyzed both software and hardware, and studied one case of the service provider. Enhancement of the server group in cloud side and multiple network accesses in client side accelerate the popularity of cloud computing technology. Being connected with various personal electric devices, cloud computing services become an essence in modern life.

\section{Introduction}

Different institutes, companies and individuals proposed more than 20 definitions of cloud computing, the concepts held similar meanings but diverse statements. But a competitively complete definition indicated by National Institute of Standards and Technology (NIST) in U.S. Department of Commerce [1] was then described as following: 'Cloud computing is a model for enabling ubiquitous, convenient, on-demand network access to a shared pool of configurable computing resources, such as networks, servers, storage, applications, and services, that can be rapidly provisioned and released with minimal management effort or service provider interaction.' In short, cloud computing is a relatively complex system.

This paper is intended to put a simple but clear insight into cloud computing technology. Starting with the basic concepts and theories of cloud computing, it explains characteristics, service models and deployment models and hardware requirements with examples and illustrates the case studies based on individual life.

\section{Characteristics of Cloud Computing}

The theories of cloud computing might be clearly understood by introducing the characteristics. Cloud Computing is a product combining almost all traditional computing technologies and network technologies, including Grid Computing, Distributed Computing, Parallel Computing, Utility Computing, Network Storage Technologies, Virtualization and Load Balance. The introduction of these technologies to cloud computing leads to a series of distinctive characteristics. According to NIST definition, cloud computing is composed of five characteristics. After classified them according to advantages and defects, the following essential characteristics would be illustrated in this paper: virtualization, elasticity and data security.

(1) Virtualization

Virtualization offers multiple virtualized resources sharing a same physical resource. NIST specification states that the technology allows hardware, such as computing units and storage devices, to be shared and software or applications to be easily migrated from one physical server to another. Thus, both hardware and software are involved in virtualization. Regarding hardware sharing, one physical device can be virtualized into multiple devices with same functions. For instance, when users try to save their files on a net-disk, such as Skydrive from Microsoft, this technology was involved because such disk is actually a virtualized hard disk of cloud computing. Concerning software virtualization, it comes from operating system (OS) virtualization. OS virtualization means that users can install multiple OSs on one computer by using virtualization software (different from 
software virtualization, a kind of software which is able to create virtualized environment) such as VMWare and VPC. After the virtualized OSs were installed, they could be moved to another computer with this virtualization software and used smoothly [2]. Cloud computing system provides many services of hardware and software virtualization on a server group or computer cluster.

(2) Elasticity

Elasticity refers to the flexibility of cloud computing related to the allocation of storage space and the dispatch of computer resource. NIST specification states that a consumer can unilaterally provision computing capabilities, such as server time and network storage, as needed automatically without requiring human interaction with each service provider. While one user requests 100 gigabytes storage space, the other user only needs 1 megabytes space, a volume could almost be ignored when compared with the demand of the former. However, in cloud computing, this technology is able to balance the space of the storage rather to limit usage of the user. In brief, elasticity of cloud computing could provide a possibility to satisfy various requirements of users.

(3) Data Security

Data security is aimed at protecting data from the destructive force and the unauthorized access. Most providers believe that cloud computing technology could guarantee a competitively high information security. Eric Farrar said: 'In fact, the complexity of security is greatly increased when data is distributed over a wider area or greater number of devices and in multi-tenant systems that are being shared by unrelated users' [3]. The open Internet and virtualization technology make the media of information transportation and storage difficult to separate physically. For instance, inside the company, the IT management department can setup a monitor port on the general router to save the network access information, including employee account number, computer name, website link, input string, and even username and password, not to mention the network outside of the company. Hence, most organizations and individuals would rather not to choose the services as such potential security risk still exists.

\section{Services and Software}

The software of cloud computing refers to system software and supporting services of cloud side and also refers to applications for the customers. According to the type of services, there are three categories classified: Infrastructure as a Service (IaaS), Platform as a Service (PaaS) and Software as a Service (SaaS). The applications are accessible from various client devices through either a thin client interface, such as a web browser or a program interface. IT companies might involve all three levels, general businesses might contact with the Software and Platform layers, but the majority of individuals might only use a part of the applications of Software layer.

Three different categories of cloud computing services would be introduced in the following. In particular, SaaS would be explained in detail because it is most closely related with our daily life.

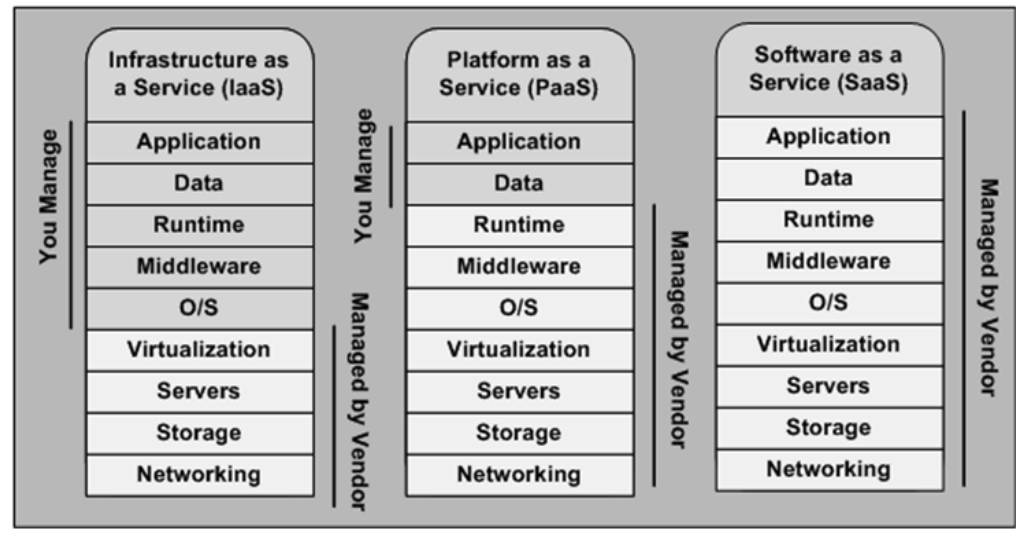

Fig. 1 Reference Models of Cloud Computing

(1) Infrastructure 
Providing processing, storage, networks, and other fundamental computing resources to the consumers, Infrastructure as a Service (IaaS) offers them to deploy and run arbitrary software, which can include operating systems and applications (see Fig.1). That is infrastructure mainly provides services including computing, network and storage for enterprises. Take cloud server as an example, companies might not purchase physical servers in data center inside the companies, but they could request cloud servers from the cloud service providers and install the operating system they might require on the cloud servers via the network connection. Based on the OS, the required applications could be installed on the servers as well as the data could be stored. And all these processes depend on a stable network communication. Therefore, computing, network and storage act as the essential components of the infrastructure layer of cloud computing.

(2) Platform

Platform as a service (PaaS) is a service model of cloud computing, in which, the consumer could create the software via tools and libraries of the provider, control software deployment and configure settings. Briefly, the consumer of PaaS could deploy consumer-created or acquired applications onto the cloud infrastructure. For example, a business could choose PaaS from a cloud provider to setup commercial website which was developed by IT department of this business. Although this is an old-fashion service model but included in the scope of modern cloud computing.

(3) Software (Applications)

Individual users might directly use the applications served by cloud providers, rather than to deploy their application. Software as a service (SaaS) provides to the consumer is to use the provider's applications running on a cloud infrastructure. 'Find My iPhone' of iCloud (from Apple Inc.) serves a good example to explain this concept. iPhone or iPad users could install 'Find My iPhone' application on the smart device(iPhone or iPad) from App Store. After a user gets the Location Services ready on his/her iPhone, it could communicates with GPS (Global Position System). And GPS will provide the location information to the iCloud. When the user tries to check his/her iPhone with "Find My iPhone" application, the application will query the data from iCloud to show the location information to him/her.

\section{Hardware Requirements}

Hardware requirements in cloud computing includes the requirements of both cloud side and client side. Cloud side refers to the service quality from providers of cloud computing, and client side means the performances of different devices which connect to the cloud computing system and utilize its resource.

(1) Cloud Side

Hardware requirement on cloud side is not how new the technology on hardware design is required, but how large the number of computers involves. Actually, obtaining the hardware information from cloud computing vendors is not a simple task. For an increasingly competitive business in IT services market today, manufacturers are not willing to release their own hardware deployment information as trade secrets. However, a researcher estimates that Amazon Cloud Services is using at least 454,400 servers in seven data centers around the globe [4]. As above, mentioned in section 2, cloud computing is a combination of traditional network technologies, computing devices and virtualization.

(2) Client Side

Network support of client side appears to be a significant factor since cloud computing heavily depends on network. Heterogeneous devices, such as smart phones, tablets, laptops, and workstations, can access cloud computing system by using multiple network accesses without location limitation. Furthermore, the improvement of network technique enables users to multiply their choices to access cloud computing, such as cable network, WIFI, GPRS and WCDMA. For example, Lenovo T420s computer, a new version laptop, not only provides RJ45 port (a cable network port), WIFI adapter and Bluetooth device to connect the network, but also contains a 3G mobile broadband device and a SIM card slot, so that the users could access to network anywhere with a 3G SIM card installed. With a 
variety of network support, these network technologies promote the popularity of cloud computing to some extent.

\section{Case Studies - iCloud of Apple Inc.}

Comparing with the products of other providers, the iCloud might be burgeoning and minimized cloud computing services, because it only provides the services for its own users to purchase the personal electronic products [5]. Unlike Amazon and other manufacturers aiming at commercial businesses, the iCloud might be closer to the individual life.

(1) Software

With iCloud, customers can automatically download new music purchases to all their devices on iTunes. They can also access media programs brought from any of their devices via wireless network and without syncing [6]. For instance, since the devices are sharing the same account, a user who bought a song Take a Bow by Rihanna on his/her iPhone with his/her iTunes account could also download this song on his/her iPad. For most users, it is a surprising experience when they play one song simultaneously on all of their devices.

Documents and presentations can be created and updated with iCloud on iMac, iPhone, iPad, and iPod touch. Its customers might not have to handle the documents in a complex file system or remember to store them to a special disk space. And the documents with all the latest edits stored in iCloud could automatically check them out everywhere the user can get iOS devices online. In result, users can create a memo on Mac and make edits to it on iPhone, or write draft on iPod touch and finalize it on iPad. Although the similar services can be found from Microsoft and Google, it could be an improvement of iCloud to Apple.

(2) Hardware

As above (Section 4), the hardware of client side could be mainly discussed this paper because impact of cloud side on individual life might not be significant. In client side, network support of iOS device appears a variety of choices (Table 1). All listed devices in the table supports WIFI and Bluetooth (third software required) network. And, providing 3G/4G network support, iPad and iPhone can access iCloud everywhere covered by the mobile signal of communication carrier.

Table 1. Network Support on iOS devices

\begin{tabular}{|c|cccc|}
\hline & iMac & iPad & iPhone & iPod touch \\
\hline WIFI & Y & Y & Y & Y \\
Cable Port & Y & A & A & A \\
3G/4G & N & Y & Y & N \\
Bluetooth & Y & Y & Y & Y \\
\hline
\end{tabular}

$\mathbf{Y}=$ Yes, available inside; $\mathbf{A}=$ Available with extra device; $\mathbf{N}=$ Not available

Offering increasingly new multimedia services on iCloud, Apple might enhance the performance of inside cameras by continually producing new devices. For example, with iPhone 4S, a customer can take high-quality photographs anywhere and share them immediately on Photo Stream in iCloud with his/her family and friends.

(3) Prospect Impact

According to information on Apple website, several new features, such as "Find My Friends" and synchronized web pages on all devices of a user, will soon land on iCloud. Find My Friends could be a method to share locations with friends and family. And Safari on iPad, iPhone, iPod touch, or Mac of a user could save the web pages on iCloud to show him/her the same page when he/she opens it on other devices. Therefore the user can get caught up on any device, even when he/she cannot access to the Internet. 


\section{Conclusion}

It is certain that cloud computing will continue to impact on individual life, but common people are still puzzling to precisely answer what the cloud computing is. In this paper, by explaining the theories with examples and illustrating case studies, this paper gives a very explicit panorama of cloud computing. This technology is increasingly impacting on individual daily life. Introduction of the essential characteristics result in the theories to be comprehensible. Classifying the services gives a schema of infrastructure, platform and software aspects. Knowledge of hardware both cloud side and client side shows that cloud computing technology heavily depends on network support. Case studies related the individuals reveal an increasing belt between cloud computing and daily life. iCloud as burgeoning cloud provider offers users more and more new features because Apple possesses an enormous user group using personal electronic products. Those client devices as cloud ladders accessing to cloud service have gradually become a part of daily life.

Acknowledgment. This work is partially supported by the Liaoning Provincial Department of Education Science Research general project under Grant No. L2014496.

\section{References}

[1] Mell P. \& Grance T. The NIST Definition of Cloud Computing, National Institute Standards and Technology, U.S. Department of Commerce, Number: 63, NIST Special Publication 800-145(2011).

[2] Marshall D., Reynolds W. A., McCrory D. Advanced Server Virtualization: VMware and Microsoft Platforms in the Virtual Data Center, Auerbach Publications (2006).

[3] Farrar E. Is Fuji Really a Cloud? [online], Available from: http://iablog.sybase.com/efarrar/ [Accessed Sep 2015].

[4] Meier J. D. Reference Models, Reference Architectures, and Reference Implementations [online], Available from:

http://blogs.msdn.com/b/jmeier/archive/2011/02/16/reference-models-reference-architectures-a nd-reference-implementations.aspx [Accessed Aug 2015].

[5] King, R. Cloud computing: Small companies take flight, Business Week [online],Available from: http://www.businessweek.com/technology/content/aug2008/tc2008083_619516.htm [accessed Jul 2015].

[6] Apple iCloud Overview [online], Available from: http://www.apple.com/icloud/features/ [Accessed Sep 2015]. 